\title{
People and Systems - Creating Networks of System Leadership and Practice
}

\author{
Steve Keyes, Organisational Development Lead for Leeds UK \\ Manraj Singh Khela, Head of Partnerships, Health \\ Partnerships Team Leeds UK \\ Frances Storr, Director, Levati, Bradford UK \\ John Walsh, Organisational Development Lead, \\ Leeds Community Health NHS Trust UK
}

\begin{abstract}
Our health systems are under pressure from increasing demands within a challenging and changing landscape. There is a need for new approaches that are joined up, relational, co-created and co-delivered. Without them there is a risk of poor experiences, a worsening of health and social outcomes and inefficient use of increasingly scarce resources. This paper reports a move towards a system leadership culture with an approach and a methodology for creating that. This requires people from different organizations with different cultures and governance to work together as if they were one organization.
\end{abstract}

This paper focuses on a major Organizational Development initiative in the city of Leeds, England to create the conditions and framework for such an approach. It reports on a methodology for creating a community of system leaders who work across the system for the benefit of the people of Leeds. A complementary aspiration of this paper was to propose a new field of theory - Dynamic System Theory - which is a derivation of Organization Development and which would benefit from further research.

One of the key achievements of this work has been an increasing distributed leadership across localities which is the key to the leadership of a complex evolving system. This has focused on people, communities and relationships, discovering common ground and connection to a bigger aim.

Key words: organization development, systems theory, system leadership, networks

\section{Introduction}

Health care systems across the world are examples of systems under pressure; struggling to cope with increasing population levels, technological developments, funding problems, rising expectations, and increasing workloads. In the UK people are living longer and as a result there are more people with complex health needs which are met by a range of providers, some public and some non-statutory services. A UK Parliamentary report Integrated Care: Organisations, Partnerships and Systems (House of Commons, 2018) proposed that:

If these services and sources of support don't join up, don't share information, are not coordinated and fail to put the individual front and centre then this can not only result in a poor experience, but risks health problems escalating and an inefficient use of increasingly stretched resources. 


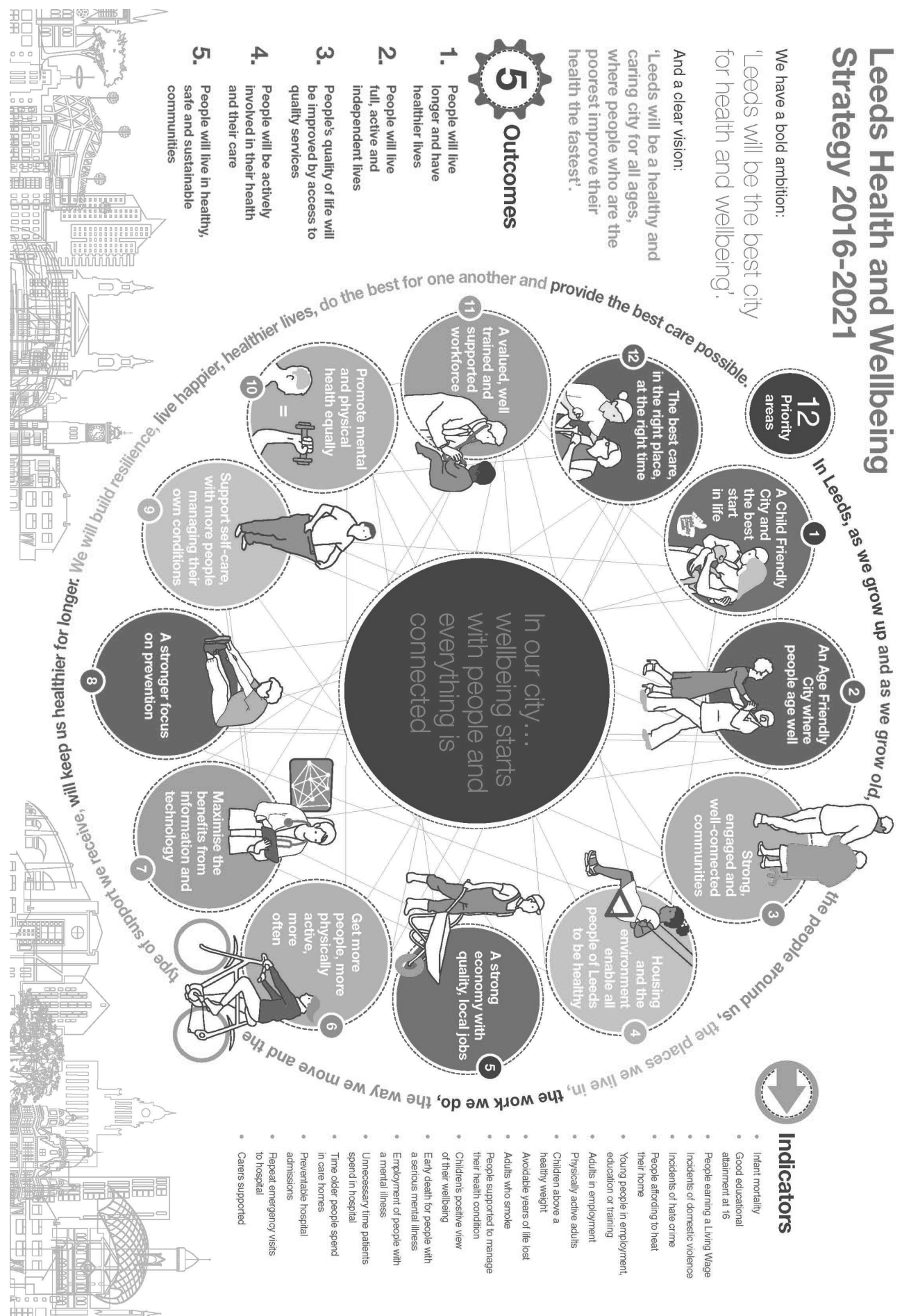

Figure 1: The Leeds Health and Wellbeing Strategy 2016-2021 
Also in 2018, the Care Quality Commission (CQC) reviewed 20 health and care systems in England with a particular focus on people aged over 65 (CQC, 2018). Even though they met hundreds of dedicated staff who are committed to providing the highest quality of care possible, they found that the health and social care system is fragmented and organizations are not always encouraged or supported to collaborate. They concluded one of the key ingredients for effective system working are "strong relationships at all levels characterized by aligned vision and values, open communication, trust, and common purpose" (CQC, 2018). Chief Executives and Senior Managers from across health and social care in Leeds were brought together to identify what problems exist and think about and support what organizational development interventions the city of Leeds should work on. One of the key actions agreed by the group was the development and embedding of a system leadership culture across Leeds.

This paper focuses upon a major Organization Development (OD) initiative to take this agenda forward. It describes the approach taken to do this, the HRD interventions introduced and provides a critical reflection upon this work to date. The work discussed in this paper is work in progress. Nonetheless the work is applicable to any sector where multi-dimensional and stakeholder engagement is critical. At one level the work represents an impactful HRD intervention for participants vis à vis bringing people together from organizations across the city to create system change. At another level it is evidence of how OD can address complex challenges beyond organizational and system boundaries. Indeed, it alerts us to the directions OD may need to take in contexts which require suspension of 'normal' or 'conventional' intervention strategies. It is suggested that an evolution of the field of OD is needed for work of this nature and Dynamic System Development (DSD) would be a more accurate description of what is involved. Further work is required to define and develop this more clearly.

The paper unfolds as follows. First, we provide more detail on the context of the OD initiative on the City of Leeds; the background and political challenges when working across complex systems and multiple stakeholder interest. This is followed by explanation of what we mean by system leadership and system practice. A discussion of the design and deployment of the HRD workshops that commenced in 2017 and continued through 2018 follows. We then reflect critically on the work to date - beginning with an assessment of impact before thinking more widely about system leadership in the context of complex change. Conclusions and implications for practice complete the article.

\section{Context}

The Leeds Health and Wellbeing Strategy provides the context for the OD interventions discussed in this paper. The strategy is captured graphically in Figure 1.

The features and characteristics of this Strategy create opportunities for the development of stronger ties and relationships; allow challenging, difficult and often intricate conversations between Chief Executives, Senior Managers, and other key stakeholders. Leeds has a strong strategic partnership Board called the Leeds Health and Wellbeing Board. This Board is supported by the Partnership Executive Group - PEG, where Chief Executives from Health, Social Care, and Third Sector organizations meet regularly to discuss their partnership working, priorities for the City and ways of working. This group identified three priority areas for OD to focus upon:

- Supporting and defining what good leadership looks like in an emerging new system. 
- Support an enabling culture that supports risk.

- The development of a clinical/care model for the city that is supported, facilitated, and transformed.

Any local Health and Wellbeing Strategy cannot prosper in isolation from national policies and practices. In this case the National Health Service (NHS) Long Term Plan (2019), and the soon to be published green paper on social care are important contextual elements. The NHS Long Term Plan (National Health Service England (NHSE), 2019) describes a model of multidisciplinary integration through expanded primary care teams based on neighbouring GP practices that work together typically covering 30-50,000 people, known as Primary Care Networks (PCNs).

For example, the NHS Long Term Plan states the following as an aim:

Breaking down traditional barriers between care institutions, teams and funding streams so as to support the increasing number of people with long-term health conditions, rather than viewing each encounter with the health service as a single, unconnected 'episode' of care (NHSE, 2019).

Such contextual factors make the work of the OD practitioner complex, requiring credibility, trust and an ethical approach in both formal and informal discussions. These same principles had to be embodied in the way the work was designed because 'the process you use to get to the future is the future you get' (Heart of the Art, n.d.). In practice it is very difficult to connect everything. Where relationships exist, people work across boundaries well but in a partnership as complex as the one in Leeds, the breadth and depth of relationships are patchy. Often the governance, processes, cultures, and pressures of work encourage people to work within their own organization rather than collaborate. They have different targets and expectations and are inspected by different bodies. For example, the hospitals are accountable for A\&E targets but the other partner organizations all do things that have an impact on that target. Shifting their behaviour to be supportive of the $\mathrm{A} \& \mathrm{E}$ target risks not meeting their own organizations targets.

\section{System Leadership and System Practice}

In this context, the system in question when we talk about System Leadership is the health and social care system in Leeds. This includes general practice and broader primary care, social care, local authority, third sector, community health, hospitals, academia, independent sector, commissioners, community groups, and citizens finding new ways to hear, understand and work together. System leadership does not relate to a person's hierarchical position but to a way of thinking, being, and behaving. People can be leaders from any place in the hierarchy or from any role. System Leadership is a theory, a practice, and a presence.

Critical theoretical influences for our work were drawn from both the literature on leadership and that of systems and change. It seems increasingly helpful to see organizations as complex evolving systems (see for example Stacey, 2003). Complex evolving systems have a number of characteristics. They survive best when they are highly interconnected and from those interconnections comes an emergent order that could not have been predicted from the behaviour of the components. They survive and thrive when they are at the edge of chaos or far from equilibrium, which is a challenging space for most human beings. Because of the sensitive dependence on initial conditions, small changes feedback on themselves and they are radically unpredictable in their behaviours. Further, there is no single unified 'Theory of Complexity', 
but several theories arising from various natural sciences studying complex systems, such as biology, chemistry, computer simulation, evolution, mathematics, and physics. This includes the work undertaken over the past four decades by scientists associated with the Santa Fe Institute (SFI) in New Mexico, USA, and particularly that of Kauffman (2000) Holland (1998), Waldrop (1992), and Gell-Mann (1994) on complex adaptive systems.

Leading in a complex system therefore requires a very different approach and we draw on the influential work done by the Kings Fund for example (Fillingham \& Weir, 2014). When a system is at the edge of chaos, a healthy place for it to be, the discontinuity and unpredictability means one cannot extrapolate from the past in order to decide what to do in the future. Instead what is needed is: pattern spotting; helping the system to be interconnected and to see itself; and experimentation followed by paying attention to the intended and unintended consequences. The leader's role is to create a container of bounded instability, hold people's anxieties, and create a climate from which helpful things will emerge. Importantly, post heroic ideas have emerged emphasizing the value of more collaborative less hierarchical practices (Collinson, 2008; Kings Fund, 2011).

The practice is a style of leadership that creates highly interconnected systems with a sense of purpose. Leading across complex interdependent systems of care is a new and different role that needs to be undertaken alongside the already difficult task of leading successful institutions (Fillingham \& Weir, 2014). Because systems thinking (and actions) puts the emphasis on connections rather than things or individuals this means holding a focus on and having skills in developing meaningful relationships. It links strongly with a person-centred view and working with people.

The Health Foundation (2016) describes a person-centred view as comprising four principles of person-centred care which as system leaders we need to work together in:1. Affording people dignity, compassion, and respect; 2. Offering coordinated care, support or treatment; 3. Offering personalized care, support or treatment; and 4. Supporting people to recognize and develop their own strengths and abilities to enable them to live an independent and fulfilling life. It is crucial that as system leaders, we are person centred at all times.

The presence is: being open, authentic, and displaying integrity, because that creates strong relationships and connections; a preparedness to relinquish control and be open to possibilities whilst at the same time being highly purposeful; able to see patterns and help the system see itself. At its core it is the emotional connection we make in our work and making a difference to people's lives in our communities. Strong ties facilitate trust, motivation, and commitment; relationships people have with teams and individuals are crucial for those leading work within and outside of a team or organizational silo. Thus, one of the aims of our work was to model system leadership in the way the intervention was designed and delivered; to be a fractal of the whole. With this in mind, a vision and a working definition of system leadership was used to anchor the intent. The vision of the intervention was 'to work as an interconnected city to be the best city for health and wellbeing,' strongly related to and connected to the Leeds Health and Wellbeing Strategy as previously described. The purpose was to create a community of system leaders who "infect" others with system leadership as a way of being. The design principles were:

- Continue to grow a connected community which has people of Leeds at the heart of everything we do. 
- Bring our partnership principles alive and embed them in what we do and in our services.

- Be clear about what it means to be a Leeds city leader, to embody local population leadership.

- Grow and develop as leaders ourselves.

- Where good things are happening, amplify them.

The working definition of system leadership was a wave of system leaders, coming from a range of levels and roles, who work beyond the boundaries of their own organization to co-create the best health and wellbeing outcomes with the people of Leeds. It requires:

- An awareness of how systems work (they are not machines).

- Courage to step into the unknown, take responsibility, and work in ambiguity.

- A focus on relationships.

- Preparedness to work for the whole system, maybe even at the expense of one's own organization or personal interests.

\section{Design, Development and Deployment}

This work was commissioned by the city-wide OD Hub and led by three of the authors, Steve Keyes, John Walsh and Manraj Singh Khela. The Hub is a self-organizing system and depends on relationships, inter-connectivity, and a commitment to support the city to support itself. The OD Hub is simply a partnership neutral physical space (a room) dedicated as a creative meeting place, connecting and supporting people from across the system. Colleagues bring challenges, problems, thoughts, ideas, opportunities to be discussed. There are no boundaries or agendas, people come along as an open space for debate, thinking and notions of what may be, with a focus on coaching and action. The aims of the OD Hub are: to map the system so people can see how the various pieces of work they do connect with each other; to connect people so they can better serve the community; to model a way of being and create viral change.

The OD Hub had been asked by the Partnership Executive Group (PEG) to craft an intervention centred on OD priorities for the city. The design and subsequent development focus was to bring these areas together collectively under our working definition of System Leadership. The task was substantial considering that approximately 57,000 staff work across the health and

care sector in Leeds. With that in mind the OD Hub approached the National NHS Leadership Academy for support, which was granted in November 2017 in the form of consulting support, Frances Storr and Kev Wyk (see also Frances Storr, author details).

A key design principle was to create something specific to Leeds which strongly related to communities. Recognizing that reasons why OD programmes may not work is that they are not always aligned with existing developments or people, are not embedded in service cultures or have no mechanisms for growth and spread. The intention was for the work to directly enable the wider strategy direction, and underlying delivery plans; link to current organizational leadership programmes/actions; and bring together a 'community' of those who do system leadership work. The work, by its very nature, would mirror, reflect, and model the supportive joined up systems that are being developed. 
The key phases of the work were:

1. Connecting with the system.

2. Day one workshops — connecting more of the system with itself, building relationships, connecting to the vision.

3. Staying connected to the Partnership Executive Group (PEG) and each other.

4. Day two workshops - moving to action.

Exploratory conversations were set up with people across health, social care, and the third sector from a variety of hierarchical levels but mostly from Chief Executives and Senior Leaders.

The conversations centred on three main interview questions:

1. What do you understand by systems leadership?

2. How does it show up in your work?

3. How do you think we are doing in Leeds?

The dialogue surfaced a number of key themes:

- The resounding message from everyone was that system leadership is all about relationships.

- Where strong relationships existed there were exemplar pieces of work utilising system leadership (see Case Study 1).

- Relationships were patchy and even the most systemic thinkers at some point in the conversation would say "they", in a slightly disparaging way to refer to others who aren't in "our group" - The implication being that "they" don't fully understand "us' or "what we do'.

\section{Case Study 1}

A group of 35 people from community and acute services, local authority, primary care, and patient representatives were brought together to design a care pathway for younger people with mental health needs. The aim of this was to prevent admissions into hospital and the new parts of the service could be led by multi-disciplinary teams. The design was done over three days and is now successfully working in the localities.

Case Study 1 gives an example of how a system can come together to resolve very practical problems that persist by being adaptive and collaborative whilst being person centred. It requires recognition of what the problem is and then creating a coalition of willing participants to come together outside of their 'normal' working roles to consider and act on behalf of the system and ultimately for the person or citizen.

A sense of 'they' is common human behaviour and the 'fundamental attribution error', (Ross, 1977) and work on in-groups and out-groups, (Tajfel, Billig, Bundy \& Flament, 1971) help us understand where it comes from. In addition, organizations have different cultures and targets with different expectations of their staff. The combination of these structural issues with the 
social psychology dynamics of 'in-groups' and 'out-groups' creates powerful forces against integration and collaboration.

Setting the ambition to grow a connected community who have people of Leeds at the heart of everything they do, a key step was to identify who to invite. This was done by asking the question "who do you know who works in a system leadership way?" i.e. "they are working beyond the boundaries of their own organization to deliver the best health and wellbeing outcomes for the people of Leeds?" and recognizing and looking for people who showed an awareness of how systems work and act with courage to step into the unknown, take responsibility and work in ambiguity.

The grapevine provided plenty of names and validated thinking that leaders in organizations do indeed span and move beyond hierarchical boundaries, even when it is difficult to do so. Initially we invited three groups of 40 to 50 people representing the spectrum of the system. GPs, Practice Managers, Allied Health Professionals (AHPs), Mental Health Nurses, and Social Workers joined us for the events, as did other people from the NHS provider organizations (NHS organizations that provide services), from the City Council, local Universities and from the third sector (non-governmental and non-profit-making organizations or associations, including charities, voluntary and community groups, co-operatives, etc.).

\section{Workshop 1}

In spring of 2018 we ran our first three one day workshops for each of the mixed groups. The objectives of the day were:

1. Reconnect with the shared understanding of the city's ambition and vision.

2. Understand how our individual work contributes to the overall city ambition.

3. Share stories of system leadership i.e. when people have taken responsibility for outcomes and worked across the system.

4. Develop shared understanding, and co-ownership, of what it means to contribute to leadership in Leeds.

5. Develop connections and relationships between people - to begin to form a system leadership community.

6. Raise awareness of the collective power in the room.

Relationships were at the core of the design. In other words, repeatedly asking people to bring their authentic self and not just show up as job holders or a named position. Goffee and Jones's (2006) work on what followers look for from leaders showed that people want to follow a human being, not a suit, and bringing one's authentic self is key to building trust and inspiring followership. The premise was that everyone in the room, whatever hierarchical level, was a leader. Facilitators did that too, role modelling showing their authentic self in the room and asking people to talk to each other about why they do what they do. There was a level of intimacy in the conversation that created honesty which in turn led to a depth of conversation and building of trust. By connecting people with each other they became more aware of each other's worlds, how their various parts of the system impact on each other and got in touch with their own passion for the work they do. The intention was to connect them to each other and to the bigger task in the city, mobilize their energy and enable them to solve problems together. 
In addition to building a community of system leaders, one of the core purposes was to inspire. This was done in a number of ways including familiarising participants with the Health and Wellbeing vision that:

Leeds will be a healthy and caring city for all ages, where people who are the poorest will improve their health the fastest.

We brought that to life in the first workshop and located the events in community venues in areas of the city with significant health and care issues. Whilst subtle, the venues brought some focus of attention and debate as well as creating the conditions for the second workshop where more focus would be put on the most deprived areas of Leeds.

The focus on people's health and being was the centre of why system leadership matters; creating spaces where people connect and work together and allowing a way forward to emerge. Other design concepts included storytelling. Denning defines a story in its broadest sense as anything told or recounted; more narrowly, and more usually, as something told or recounted in the form of a causally-linked set of events; account; tale; the telling of a happening or connected series of happenings, whether true or fictitious (Denning, 2000).

As such, stories give sense to things and events and represent norms, experience, and explanations of reality. Stories powerfully convey norms and values across generations within an organization or system. In our work we were conscious of how to develop the trust and commitment by revealing our personal stories which can expose one's own passion and commitment, as well as signal one's trust in and willingness to be vulnerable to others.

We deliberately framed conversations in a positive, strength-based way; inviting people to tell each other about moments when they felt they were demonstrating system leadership. This was in the spirit of 'catch ourselves doing it right'. The intention was to spread those stories around to inspire others as well as increase self-efficacy by giving affirmation and recognition for those moments.

We introduced a Google + social media site that was a closed community for people who attended the workshops and called it Leading Leeds. We posted materials and articles of relevance and invited participants to do the same. We also invited anyone who was brave enough, to post a one-minute video of themselves telling their system leadership stories so that others could see and be inspired. Over time this will build as an important archive, through which a sample of system leadership stories, people's enthusiasm, dedication and commitment for working across boundaries in Leeds will be showcased.

In the afternoon we created an Open Space for people to gather and work on the issues that they identified we should be working on together. Open Space Technology is an approach for hosting meetings developed by Owen (2008) after he had attended conferences and found that the most valuable conversations were the informal ones that happened spontaneously. In Open Space the topics for discussion are determined and organized by the people attending the meeting and there is a very specific process for creating a self-organizing system.

A summary of where these conversations got to and who was in them was posted on the Google + site. The philosophy with these was that participants were not reporting back to the 'organisers', but making the information available to each other who then in turn could build on the conversation. Figure 2 provides an example. 


\begin{tabular}{|c|}
\hline Topic \\
\hline $\begin{array}{c}\text { 6 Priority Neighbourhoods - } \\
\text { Clyde's, Lincoln Green, Newell's, Recreations, Boggart Hill, Beverley's and Stratford's. }\end{array}$ \\
\hline Convenor \\
\hline Sarah Kemp \\
\hline Who else was present \\
\hline Karen Otway, Ruth Corneilson, Kulvant Sandhu, Helen Kemp, Caroline Schonrock \\
\hline Summary of where we got to \\
\hline $\begin{array}{l}\text { - LCPs, Learning Cafés e.g. LS14 Trust. } \\
\text { - Community connections, community development, wider determinants of health, use community } \\
\text { connections to help health find out what people would like and need. } \\
\text { - Housing Conditions, working with partners to change service for users. Trying to change culture for } \\
\text { years of deprivation, need to work with staff and service users, we need to ensure resources are used } \\
\text { properly, e.g. unnecessary home visits and working with, local knowledge key about cases. } \\
\text { - Leg clubs tackling isolation, could be jobs clubs etc. - Adult Walking Bus. }\end{array}$ \\
\hline What next? \\
\hline $\begin{array}{l}\text { - What can we offer - community matron and therapist going to cafés to see what potential is for } \\
\text { 'working with' community and what the community needs and respond. } \\
\text { - Don't have a set agenda, allow staff to be brave and get out there to connect and build relations with } \\
\text { the community and the agencies such as third sector that are already present. Could be ESOL, job } \\
\text { clubs, what does the community want? } \\
\text { - Shift resource to these priority areas. } \\
\text { - Difficult conversations and decisions to be made re-funding/resource. } \\
\text { - Local relationships - you know the police, you know the doctor, you know the head teacher etc. } \\
\text { - Have champions for safeguarding other issues, social prescribing. } \\
\text { - Links with Third Sector. } \\
\text { - Prevention of falls/health issues. } \\
\text { - Hospital prevention, home first - change risk attitudes. }\end{array}$ \\
\hline What can you do \\
\hline $\begin{array}{l}\text { - Debt workers, money and benefits advice, befriending, confidence building. } \\
\text { - Connect } 4 \text { year olds with older people, connect schools and care homes. } \\
\text { - Support the changes to Leeds Directory and 'what's going on in Leeds' and make it live, could it pull } \\
\text { info from social media. } \\
\text { - Can we target children in school, early years, to try influence change in family cycle? } \\
\text { - Seacroft are consulting at the moment to see what service users would like in terms of those who are } \\
\text { housebound, could they implement a 'leg club' model, or something different, pulling in feedback at } \\
\text { the moment, they have never consulted like this before. } \\
\text { - Need to do more consulting, not be reluctant about going into priority neighbourhoods, what are } \\
\text { the barriers, being safe etc. How can we engage, have those 'better conversations'. Look at the } \\
\text { whole person, mental health issues, police, ASBU, health, education, all need to be joined up and } \\
\text { communities enabled and resourced to tackle complex circumstances together. }\end{array}$ \\
\hline
\end{tabular}

Figure 2: Open Space Feedback sheet

At the end of the day participants were invited to make a commitment. We had already acknowledged that system leadership takes courage and we invited people to name an act of courage, large or small, that they were prepared to do. We were inspired by what came back, both by the nature of some of the commitments, e.g. moving towards people or issues that had been avoided, and also by the way in which they were spoken — with heart. 
The very first day one workshop was a partial success. We realized that we had tried to take the group too far too quickly into a session that was rather different to what they were familiar with. The design evolved for groups two, three, and beyond.

Subsequent feedback from participants was that the sessions were very different to the events they are usually invited to and what they were expecting - in a good way. In part this was due to mix of professionals, differing organizations, and hearing about vulnerable people they had encountered in their jobs. They were enthused and inspired during and after the sessions. They felt a real sense of connection, shared purpose, and collective energy for positive action.

From the beginning of the work staying connected to the leaders of the partner organizations in the city was key. The system leadership work was designed in such a way that it wasn't led from the top down. The request of the Partnership Executive Group was not to direct this from the 'top down' but to ensure there was congruence between what they as leaders were asking their teams to do and the work the intervention was delivering. We also asked PEG to pay attention to what they were role modelling both collectively and individually and to support leaders who engage in the work. One of the ways of keeping this work connected to the broader strategic challenges was to ask participants to have a conversation with their executive or PEG member after the workshops and, in parallel, ask PEG to meet with participants after the workshops. These conversations happened to different extents but when they did, they produced new ideas or a positive change in thinking and behaviours.

In the day one workshops, acknowledging that a community doesn't get built in a day, facilitators committed to bringing people together for a second day two months after their first session. This allowed time for reflection and making further connections with others and in particular PEG members.

\section{Workshop 2}

The objectives for our second series of workshops were:

1. Reconnect with each other and update on what's being going in the system since the first workshop.

2. Stimulate people with what's possible.

3. Continue to develop connections and relationships between people - build our system leadership community.

4. Raise awareness of the collective power in the room and mobilize energy.

5. Help each other to tackle our challenges in our work in our bit of the system.

6. Raise awareness of six priority areas of deprivation in the city.

The first half was a reconnection with each participant and further sharing of experiences and stories of people that they work with in their part of the system; presenting data and stories around the most deprived areas in Leeds. Many of these deprived areas are amongst the poorest neighbourhoods in the country. The dissonance between Leeds as a thriving economy and areas of intense deprivation and growing inequalities was apparent. In the afternoon we moved to a series of practical actions that could be taken to support the work in our communities. We used 
the framework from Asset Based Community Development (ABCD) which switches attention from deficits to amplifying strengths, Figure 3.

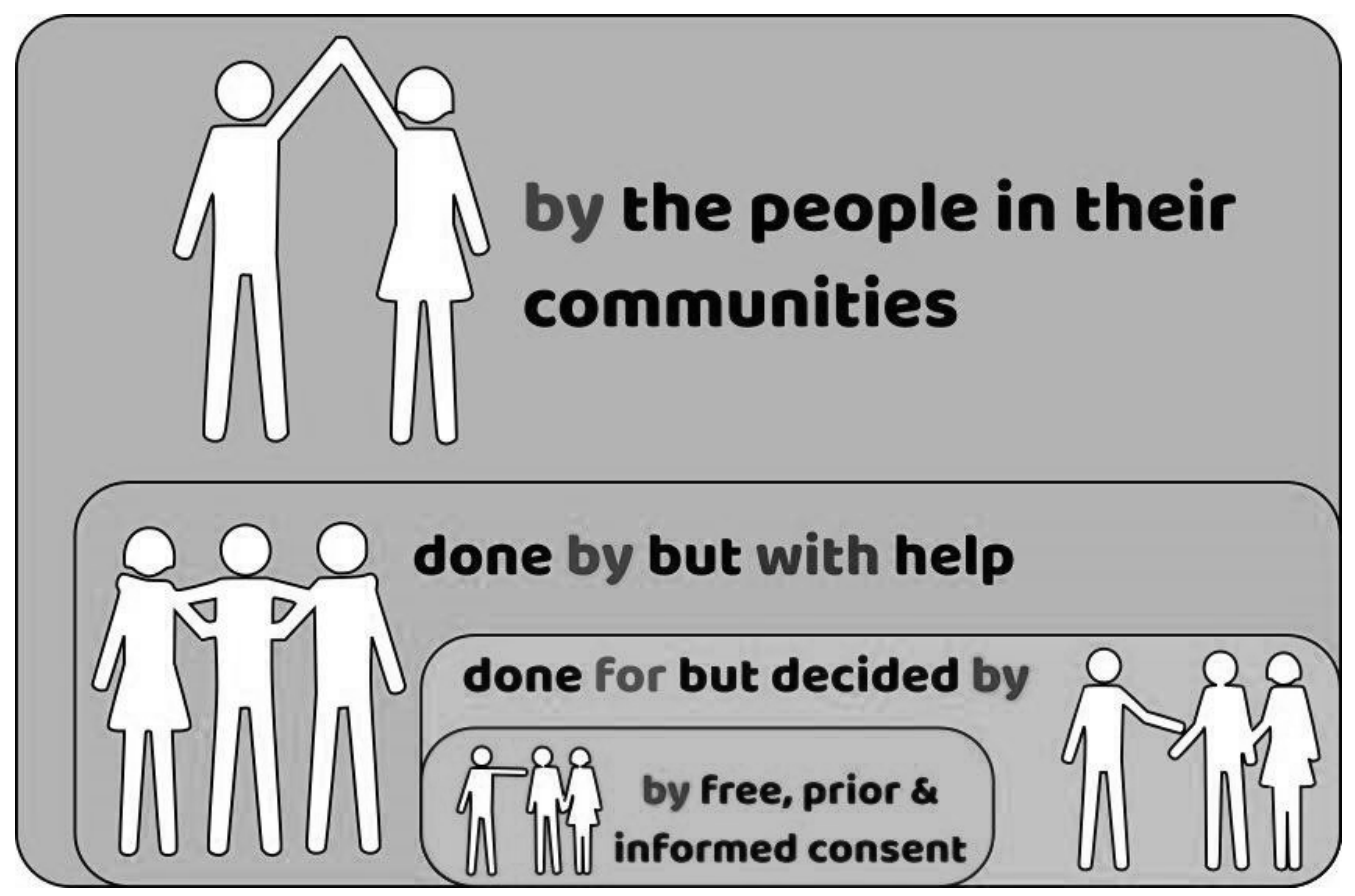

Figure 3: Asset Based Community Development

ABCD is built on four foundations (Kretzmann 2010)

1. It focuses on community assets and strengths rather than problems and needs.

2. It identifies and mobilizes individual and community assets, skills, and passions.

3. It is community driven — 'building communities from the inside out'.

4. It is relationship driven.

One of the powerful elements of the day was "Wants and Offers". 'Wants', being things that someone needs support or advice with and 'offers' being something that a person can provide or share with others. The session was designed as a self organizing marketplace. This quid pro quo exchange allowed people to see the extent of wisdom in the room and the extent to which people were willing to help one another. Whilst hesitant about what kind of use participants would make of this, people embraced the opportunity and created a marketplace of various people approaching each other to respond to the wants and offers they had posted.

\section{Impact}

At the time of writing this article over 200 people had participated in each of the two days. A further 200 are expected to go through the sessions set out for 2019. Our evaluation to date is 
based primarily on feedback from participants. Feedback from people after the events was that there was an immediate impact as well as longer lasting positive impact.

Prior to being part of the workshops, many participants indicated being overwhelmed in their work. They had heard the information about the vision for Leeds, the need for more preventative work in communities and pockets of deprivation etc. but not in a connected, coherent way. The workshop helped people to see how all of those things join up and also gave them space to think about how they and their teams can contribute. Participants described how the workshops are not like anything else they attend and they particularly valued the quality of relationships they are forming with people they wouldn't normally get to meet. Anecdotal feedback about the immediate impact of the session included:

There have been some real practical connections enabling me to solve some problems and work more effectively in the system.

Having this time out to come together with others across the system has been valuable reflective development and will enable me to do my job better as a system leader.

The two self-organizing elements previously referred to: Open Space on workshop one and the wants and offers section on workshop two stimulated people to act in a more system related way. Case Study 2, below, provides an example.

\section{Case Study 2}

The role of the Leeds Health Protection Board is to undertake the duties to protect the health of the population as laid out in national guidance. Since 2014 the Leeds Health Protection Board, chaired by the Director of Public Health, has been leading programmes of work focusing on identified emerging health protection priorities for Leeds. The Head of Public Health (Health Protection) outlined some personal as well as practical work that emerged through engaging with the system leadership work:

- A validation of the system work in health protection and adding confidence to her portfolio of work.

- Making the system work more implicit in the work and reporting to the Health and Wellbeing Board.

- A system wide partnership approach has been implemented across the health protection priorities within Leeds. An example of this includes the influenza vaccination campaign in 2018 targeting priority groups and low uptake areas in Leeds. For the first time Leeds achieved the national target of $75 \%$ uptake for flu vaccination in over $65 \mathrm{~s}$ for NHS Leeds CCG. Uptake for flu vaccination for other target groups was also higher than last year. Flu vaccination levels were also increased on last year in Leeds Community Healthcare (77\%), Leeds Teaching Hospitals Trust (80\%), Leeds and York Partnerships Foundation Trust (65\%), and Leeds City Council (911 Leeds City Council members of staff received their seasonal flu immunization — an increase of over 300 from the previous year).

The Case Study is an example of connecting the system to itself, to the vision and enabling people to join together and solve things that they can see need solving. The experience of the workshops created emotional connection, motivation and movement to action. This is the core of the work. The more that work is done in this way, the more that can be done that moves beyond the boundaries of respective organizations and to the heart of system leadership. 
At the end of each session, acknowledging that system leadership takes courage and asking people what (small or large) courageous thing they will do to model system leadership was a way to make conscious the move to action. Participants thought carefully about their responses, with some being inspired and pushing themselves to be even more courageous when they heard what their colleagues were committing to.

Examples of acts of courage were:

- Explore how we can support others to make sure of a voice/awareness of advanced decisions and end of life discussions.

- To contact 3rd sector partners to support system of community development.

- Have a conversation with a Hospital Consultant around a current problem that's causing a barrier to thinking 'home first'.

The real strength of this work is that it brings together people, most of whom would never meet to talk about their city, stories, and hopes, to create new relationships and connect people to a common vision which transcends functional or organizational boundaries. Our summative evaluation is that there are three common features that emerged as a result of the work done to date:

- People discovering their answers in the wider system they were meeting. Often observing participants wanting someone to talk to about an issue and someone else from another part of the system would spontaneously connect with them to share links, resources, and ideas.

- People's vision and mindset moving to a more city perspective but with a real appreciation for the communities we serve; a sense of 'us' and 'we' together creating the future seeing and experiencing themselves as more of a system across a city than a silo.

- Creating a safe space for participants to talk about what matters to them and naming gaps in systems with a needed new focus on marginalized communities, third sector and children's services.

\section{Reflection on Action}

On a practical level, there were a number of things we did to create intimacy and trust, which created a climate that was markedly different from many of the work conversations about these same issues. An example was asking participants to talk to each other as human beings not just job holders, and modelling that as facilitators. The intention was to bring our authentic selves, to encourage them to bring their authentic selves and to create conversations that were deeper and more open than many of the conversations they have at work. We supported this by designing the room very specifically to enable intimate conversations i.e. no tables, small groups of four or five people and repeatedly mixing the groups. Another of the intentions was to create emergence by stimulating connections and conversations that would not normally happen. So the groupings were changed around so that people met as many participants as possible over the course of the workshops and the participants were a 'diagonal slice' of hierarchy and organization. Thirdly the intention was to inspire and to start with an assumption that people are already doing inspiring things, large and small, and they would be moved by hearing each other's stories of these. This was in fact the case although people often needed encouragement to overcome natural modesty in telling these to each other. 
One of the underlying principles was to help people connect with what they find meaningful in their work, in the belief that this releases energy. Inviting people to talk about why they do the work that they do was an important precursor to the Open Space where the principle was to trust that people know what is the work that needs to be done.

The flexibility of the facilitation team was also extremely important. For example, there was a realization that some of the design elements of the very first workshop were too far removed from what people were comfortable with and the design was quickly amended. Some judgements were made in the moment, based on the responsiveness of the group e.g. taking a risk at the end of a workshop by asking participants to write down an act of courage and then asking them to say it to the group. The size and composition of groups varied and the design had to be adjusted in flight to what was happening in the room. The learning was to push participants to be open and to take responsibility for their part without making them so uncomfortable that they did not want to participate.

As a tool for spreading the practice of system leadership and creating a movement a social media site can be a huge asset. In practice it was difficult to engage people with the Google+ site. We believe this was due to the fact there were a couple of steps to take to get into the site and also people are overwhelmed with a combination of emails and social media.

There is value in extending this reflection on action to some broader considerations about system leadership and how OD might best take forward the development of system leadership in complex scenarios such as the one discussed in this paper. System leadership is challenging both in concept and action and this work is itself a piece of system leadership. It was not always clear who to talk to or how to create energy in the system. It is not set within a formal organization structure, it is working across boundaries, enacting through the development of relationships but always with a strong sense of purpose.

Traditional formal authority is not enough to create a community of system leaders. One of the things that has made this work is the relational focus of all involved. Some were already highly connected in the system and actively used their relationships to influence. For example, getting people to turn up to the events was never going to be achieved by sending an email invitation alone. For the first events it was relationships that induced people to come and after that the reputation of the workshops helped to bring a continued stream of participants. In parallel the authors proactively developed relationships with people who could be pivotal in helping to make this happen.

The power of the workshops was a combination of connecting people to the problem and to each other, and appealing to people's values of justice and hope (Axelrod, 2003). This was especially true in the day two workshop when presenting the data on six of the most socially and economically challenging areas of Leeds. However, hope for change is not enough to make change happen. Beckhard and Harris's (1977) change equation suggests that the necessary conditions for change are a clear shared vision, knowledge of the first steps to take and sufficient dissatisfaction with the present. The intention was to increase dissatisfaction with the present sufficiently to propel people to action but not overwhelm them with it.

On its own, having an event outside of any organizational or system context will simply be an event; unconnected to its core assets and the people in which we serve. The ambition is to create a growing community through other events like this over time. This in turn connects to community action and other development opportunities provided by all organizations across the health and 
care sector. 'Folding' our activities back into an organizational context where Chief Executives, Chief Officers and others can engage further with participants from system leadership events is a way of amplifying the work. Through these connections together they have developed shared values, shared understanding, shared trust, shared ideas and shared hope that leads to shared action. The plan is to amplify and embed the work across the system.

Paying attention to our own system as a delivery team has also been key. Some of the longest conversations the design team had were at the beginning to clarify the purpose and design principles of the work. At this stage the relationships between the team and others was still developing and this presented its own challenges, not just in getting time together but also understanding each other's different world views and co create the events. What surfaced was making time to hear the different perspectives, not be dismissive of any of them and have robust conversations, especially about what the purpose and outcomes would be.

Support to the system to facilitate change and enable new types of leader to emerge is a development of Organizational Development (OD) practice. This new landscape encompasses the need to work across whole systems whilst utilising core professional skills and interventions. This evolving practice; Dynamic System Development (DSD) is an advent of the needs of the system and for the practitioner to build on the role of OD practice, from organization to whole system work. Traditional OD, which emerged in the 1960s, conceptualizes change as a planned process, moving from unfreezing to refreezing and with an emphasis on aligning various organizational elements to position the organization for future success. Dialogic OD, whilst adhering to the same underlying values and ethics as traditional OD, proceeds from a different mindset about change. The emphasis is on changing organizations by changing the conversations (Shaw, 2002). Organizations are seen as networks of relationships where actions result from socially constructed realities created and sustained by the prevailing narratives, stories and conversations through which people make meaning about their experiences (Marshak, 2013). Change emerges from disturbances that change the conversations, which shape meaning making and everyday thinking and behaviour. The intention was to change the system by changing the conversations. The focus of the interventions was not to diagnose and then agree on an action plan but to create containers which provide the right ingredients for participants to:

- Inquire together.

- Form new connections and enrich existing connections.

- Connect to the bigger aims.

- Be generative.

- Create emergence.

At times it was difficult not to get drawn back into a paradigm of diagnose, develop an action plan, execute, and measure. Questions from stakeholders often came from that world view. Conversations about evaluation are complex because they are at the cusp of two very different philosophical viewpoints. The focus is on fostering conditions that will lead to new ways of thinking and behaving and better outcomes. The intention is to unleash, catalyse, and support the multitude of motivations and ideas among participants in the service of transforming the system in the desired direction. 


\section{Conclusions and Implications}

One of the achievements of this work is increasing distributed leadership across a system, which is key to the leadership of a complex evolving system. This has come through raising awareness of people's potential to achieve things in a collaborative, cross system way and which produces increased energy, creativity, and responsibility. The necessary conditions to bring this about are: invite a diverse group, a microcosm of the whole system; connect people to a meaningful purpose and to each other; increase self efficacy; create a container and get out of the way i.e. trust people to do good work and not try to over manage. This work has demonstrated a very practical methodology for creating collaborative leadership across a system whilst highlighting possible traps and important subtleties. What has made the work successful is meticulous attention to congruence; between design principles and practice.

The starting point was to gather the most senior leaders in the city to engage and produce priorities for OD/DSD interventions, ensuring that any design and deployment was premised on the city's ambition to improve the health of the poorest the fastest and contributing to the Leeds Health and Wellbeing Strategy. Policy makers and politicians in the past have reached for structural levers in order to achieve their outcomes. Top down restructuring and reorganizing in order to improve efficiency, quality and improve services for citizens is often employed in preference to collaboration, leadership and partnership working with communities.

A complementary aspiration of this paper was to encourage thinking about a new field of theory — Dynamic System Theory — and which is a derivation of Organization Development. There is similarity in the skills required of practitioners. The profile of a competent OD practitioner (Cheung-Judge \& Holbeche, 2011) would be similar to the profile of a competent DSD practitioner. A key difference is that in system development there is no senior figure who has the ultimate say because it is a network of organizations. The collaborating organizations have no formal authority over each other and have different governance, cultures, and pressures. This requires a higher degree of political intelligence.

This is a developing field in many sectors; for example alliances that are created to deliver large infrastructure projects, and is worthy of further research to define the discipline. This is not just different ways of working but considering how, what and why brings people together to create system change in an ever changing and often turbulent context. The deep focus and nature of system leadership in this relational way helps individuals to connect with themselves and their profession. A bigger purpose is the way existentially that brings leaders together across the system to support the cities ambition and improves people's lives.

\section{The Authors}

Steve Keyes is an organizational psychologist who leads OD in the city as part of the Leeds Health and Care Plan (the partnership change programme for the delivery of the health and care aspects of the Leeds Health and Wellbeing Strategy). Manraj Singh Khela is a change management specialist who works in the Leeds Health Partnerships Team (a central team cofunded by partners across Leeds). Frances Storr is an organizational psychologist and director of Levati, and John Walsh works in OD at Leeds Community Healthcare. 


\section{References}

Axelrod, R. (2003). Terms of Engagement. Berrett-Keohler

Beckhard, R., \& Harris, R. T. (1977). Organizational transitions: Managing complex change Reading, MA: Addison-Wesley.

Care Quality Commission (CQC) (2018). Beyond Barriers: how older people move between health and social care in England, Care Quality Commission, Newcastle Upon Tyne; https://www.cqc.org.uk/ sites/default/files/20180702_beyond_barriers.pdf

Cheung-Judge, M. \& Holbeche, L. (2011). Organisation Development. A practitioner's guide for OD and $H R$. Kogan Page.

Collinson, D. (2008). Distributed and Shared Leadership, CEL Practitioner Research Projects 2007-8, Centre for Excellence in Leadership, University of Lancaster

Fillingham, D., \& Weir, B. (2014). System Leadership: lessons and learning from AQuA's Integrated Care Discovery Communities, Kings Fund, London; https://www.kingsfund.org.uk/sites/default/files/field/ field publication_file/system-leadership-october-2014.pdf

Gell-Mann, M. (1994). The Quark and the Jaguar: Adventures in the Simple and the Complex. W. H. Freeman.

Goffee, R., \& Jones, G. (2006). Why Should Anyone be Led By You? What it takes to be an authentic leader, United States: Harvard Business Review Press.

Harrison, O. (2008). Open Space Technology: A User's Guide. Pub Berrett- Koehler

Health Foundation (2016). Person-centred care made simple: what everyone should know about personcentred care, The Health Foundation, London.

Heart of the Art (n.d.), Myrons Maxims retrieved from https://www.heartoftheart.org/?p=1196

House of Commons (2018). Integrated care: organisations, partnerships and systems. Seventh Report of Session 2017-19. Report, together with formal minutes relating to the report Ordered by the House of Commons to be printed 23 May 2018. House of Commons. Health and Social Care Committee.

Kauffman, S. (1993). The Origins of Order: Self-Organisation and Selection in Evolution. Oxford University Press

Kauffman, S. (1995). At Home in the Universe. Viking

Kauffman, S. (2000). Investigations. Oxford University Press

Kings Fund (2011). The Future of Leadership and Management in the NHS: no more heroes; Report from The King's Fund Commission on Leadership and Management in the NHS; https://www.kingsfund.org. uk/sites/default/files/future-of-leadership-and-management-nhs-may-2011-kings-fund.pdf

Kretzmann, J. P. (2010). Asset-based strategies for building resilient communities. In J. W. Reich, A. Zautra \& J. S. Hall (Eds.), Handbook of adult resilience. New York: Guilford Press.

Kretzmann, J. P., McKnight, J., \& Green, M. (1997). A guide to capacity inventories: Mobilizing the community skills of local residents. Chicago, IL: ACTA Publications. Available from http:// communitymagic.org/public/A\%20Guide\%20to\%20Capacity\%20Inventories.pdf

Marshak R. J. \& Bushe G. R. (2013). An introduction to advances in dialogic organisational development. OD Practitioner, 45(1), 1-3

NHS Long Term Plan. (2019), Department of Social Security.

Ross, L. (1977). The intuitive psychologist and his shortcomings: Distortions in the attribution process. In Berkowitz, L. Advances in experimental social psychology. 10. New York: Academic Press. pp. 173-220.

Shaw, P. (2002). Changing Conversations. Pub Routledge

Stacey, R. D. (2003). Complexity and Group Processes: A Radically Social Understanding of Individuals. New York: Brunner-Routledge.

Tajfel, H., Billig, M. G., Bundy, R. P., \& Flament, C. (1971). Social categorization and intergroup behaviour. European Journal of Social Psychology, 1(2), 149-178.

Waldrop, M. M. (1992). Complexity: The Emerging Science at the Edge of Order and Chaos. Penguin 1994. 